\title{
A NOTE ON INVERSE FUNCTION THEOREMS
}

\author{
E. B. LEACH
}

In this note we use the Newton-Raphson approach to inverse function theorems. We draw natural conclusions when only a left or a right inverse to the differential at a point is available. By using a strengthened version of differential, we are able to use differentiability at a single point as the smoothness condition. Although the method has been used before (cf. [2, p. $167 \mathrm{ff}$.]), analysis books have tended to use an approach that assumes finite dimensionality of the reference spaces.

Definition. Let $U$ and $V$ be Banach spaces and $f: U \rightarrow V$ a function. A strong differential of $f$ at a point $x_{0}$ in $U$ is a bounded linear transformation $\alpha: U \rightarrow V$ which approximates changes of $f$ in the sense that for every $\epsilon>0$, there is a $\delta>0$ such that if $x^{\prime}$ and $x^{\prime \prime}$ are nearer than $\delta$ to 0 , then:

$$
\left|f\left(x^{\prime}\right)-f\left(x^{\prime \prime}\right)-\alpha\left(x^{\prime}-x^{\prime \prime}\right)\right| \leqq \epsilon\left|x^{\prime}-x^{\prime \prime}\right| .^{1}
$$

A relation such as (1) for any $\epsilon$ and $\delta$ implies a Lipschitz condition, and so continuity of $f$ in the sphere $\left\{\left|x-x_{0}\right|<\delta\right\}$. If $f$ should have a Fréchet differential $\alpha_{1}$ at a point $x_{1}$ of that sphere, it is clear that $\left|\alpha_{1}-\alpha\right| \leqq \epsilon$. The following theorem is specialized by considering $x_{0}=0$ in $U$, and by the condition that $f(0)=0$. It is evident that a more general situation reduces to this by translation of range and domain; however, the statement and the proof of the theorem are a little more complicated in the general case.

Theorem. Let $f: U \rightarrow V$ be a function such that $f(0)=0$ and $f$ has strong differential $\alpha$ at 0 . Let $\beta: V \rightarrow U$ be a bounded linear transformation such that $\beta \alpha \beta=\beta$. Then there is a function $g: V \rightarrow U$ such that $g(0)=0, g$ has strong differential $\beta$ at 0 , and $g$ satisfies for $y$ near 0 the identities:

$$
\begin{aligned}
& \beta(f(g(y)))=\beta(y), \\
& \beta(\alpha(g(y)))=g(y), \\
& g(f(\beta(y)))=\beta(y) .
\end{aligned}
$$

Any two g's satisfying these conditions are identical for $y$ near 0 .

Presented to the Society, February 25, 1961; received by the editors April 5, 1960 and, in revised form, November 3, 1960.

${ }^{1}$ We will use the notation $|x|$ or $|\alpha|$ for the norms of vectors or of linear transformations. 
Proof. For any $y$ in $V$, sequences $\left\{x_{n}\right\}$ and $\left\{y_{n}\right\}$ are defined by $x_{0}=0$ and the recursion formulas:

$$
y_{n}=f\left(x_{n}\right), \quad x_{n+1}=x_{n}+\beta\left(y-y_{n}\right) \quad(n=0,1,2, \cdots) .
$$

We define $g(y)$ to be the limit of $\left\{x_{n}\right\}$ for all $y$ such that the limit exists, and to be 0 otherwise. For all $y$ sufficiently near to $0, g(y)$ is the limit of $\left\{x_{n}\right\}$.

To prove convergence of $\left\{x_{n}\right\}$ for $y$ near 0 , consider $\epsilon>0$ such that $\epsilon|\beta|<1 / 2$ and $\delta>0$, chosen so that (1) is valid for $f$ at the origin of $U$. We prove inductively that $\left|x_{n}\right|<\delta$ and that $\left|x_{n+1}-x_{n}\right|<(\epsilon|\beta|)^{n} \delta / 2$, provided $|y|<\delta / 2|\beta|$, for $n=0,1,2, \cdots$. The bound on $\left|x_{1}-x_{0}\right|$ is an immediate consequence of (3), for $|y|<\delta / 2|\beta|$. If $\left|x_{m+1}-x_{m}\right|$ $\leqq(\epsilon|\beta|)^{m} \delta / 2$ for $m \leqq n-1$, then $\left|x_{n}\right| \leqq \sum_{m=0}^{n-1}(\epsilon|\beta|)^{m} \delta / 2<\delta$, since $\epsilon|\beta|<1 / 2$. Now we have by (3) and the relation $\beta \alpha \beta=\beta$, for $n \geqq 1$ :

$$
\begin{aligned}
x_{n+1}-x_{n} & =\beta\left(y-y_{n}\right)=\beta\left(y-y_{n-1}\right)-\beta\left(y_{n-1}-y_{n}\right) \\
& =\beta \alpha \beta\left(y-y_{n-1}\right)-\beta\left(y_{n-1}-y_{n}\right) \\
& =\beta\left[\alpha\left(x_{n}-x_{n-1}\right)-f\left(x_{n-1}\right)+f\left(x_{n}\right)\right] .
\end{aligned}
$$

The vector in brackets is essentially the vector of the left side of (1), with $x^{\prime}$ and $x^{\prime \prime}$ replaced by $x_{n}$ and $x_{n-1}$, and since these fall within $\delta$ of 0 , (1) implies $\left|x_{n+1}-x_{n}\right| \leqq|\beta| \cdot \epsilon\left|x_{n}-x_{n-1}\right|$, from which the asserted bound on $\left|x_{n+1}-x_{n}\right|$ follows by induction. The sequence $\left\{x_{n}\right\}$ therefore converges uniformly to $g(y)$ if $|y|<\delta / 2|\beta|$.

The identities $\left(2^{\prime}\right)$ and $\left(2^{\prime \prime}\right)$ are readily derived for all $y$ such that $|y|<\delta / 2|\beta|$. For $\left(2^{\prime}\right)$, let $n \rightarrow \infty$ in (3), and taking account of the fact that $f\left(x_{n}\right) \rightarrow f(g(y))$ by continuity of $f$, we have $g(y)=g(y)$ $+\beta(y-f(g(y)))$, which simplifies immediately to $\beta f g=\beta$ for $|y|$ $<\delta / 2|\beta|$. For $\left(2^{\prime \prime}\right), \beta \alpha\left(x_{n+1}-x_{n}\right)=\beta \alpha \beta\left(y-y_{n}\right)=\beta\left(y-y_{n}\right)=x_{n+1}-x_{n}$, and inductively it follows that $\beta \alpha x_{n}=x_{n}$. Making $n \rightarrow \infty, \beta \alpha g(y)=g(y)$ for all $y$ such that $\left\{x_{n}\right\}$ converges (and for all other $y$, since then $g(y)$ $=0=\beta \alpha g(y)$ ).

Each of the remaining steps of proof involves estimating $x^{\prime}-x^{\prime \prime}$ for some choice of $x^{\prime}, x^{\prime \prime}$ nearer than $\delta$ to 0 in $U$. In each case a differential relation involving $\beta\left(f\left(x^{\prime}\right)\right)-\beta\left(f\left(x^{\prime \prime}\right)\right)$ is invoked. First we prove a Lipschitz condition on $g$. If $y^{\prime}$ and $y^{\prime \prime}$ are nearer than $\delta / 2|\beta|$ to 0 in $V, g\left(y^{\prime}\right)$ and $g\left(y^{\prime \prime}\right)$ are nearer than $\delta$ to 0 in $U$, and by (1), with this choice of $x^{\prime}$ and $x^{\prime \prime}$ :

$$
\left|f\left(g\left(y^{\prime}\right)\right)-f\left(g\left(y^{\prime \prime}\right)\right)-\alpha\left(g\left(y^{\prime}\right)-g\left(y^{\prime \prime}\right)\right)\right| \leqq \epsilon\left|g\left(y^{\prime}\right)-g\left(y^{\prime \prime}\right)\right| .
$$

Application of $\beta$ to the vector in brackets leads to the relation: 


$$
\begin{array}{r}
\left|\beta\left(f\left(g\left(y^{\prime}\right)\right)\right)-\beta\left(f\left(g\left(y^{\prime \prime}\right)\right)\right)-\beta \alpha\left(g\left(y^{\prime}\right)-g\left(y^{\prime \prime}\right)\right)\right| \\
\leqq \epsilon|\beta|\left|g\left(y^{\prime}\right)-g\left(y^{\prime \prime}\right)\right| .
\end{array}
$$

The vector on the left simplifies, because $\beta f g=\beta$ and $\beta \alpha g=g$, to:

$$
\left|\beta\left(y^{\prime}-y^{\prime \prime}\right)-\left(g\left(y^{\prime}\right)-g\left(y^{\prime \prime}\right)\right)\right| \leqq \epsilon|\beta|\left|g\left(y^{\prime}\right)-g\left(y^{\prime \prime}\right)\right| .
$$

By this relation and the triangle inequality we obtain $\left|\beta\left(y^{\prime}-y^{\prime \prime}\right)\right|$ $\geqq\left|g\left(y^{\prime}\right)-g\left(y^{\prime \prime}\right)\right|-\epsilon|\beta|\left|g\left(y^{\prime}\right)-g\left(y^{\prime \prime}\right)\right|$, and since $\left|\beta\left(y^{\prime}-y^{\prime \prime}\right)\right|$ $\leqq|\beta|\left|y^{\prime}-y^{\prime \prime}\right|$ and $1-\epsilon|\beta|>0$, we obtain the Lipschitz condition valid if $y^{\prime}$ and $y^{\prime \prime}$ are nearer than $\delta / 2|\beta|$ to 0 :

$$
\left|g\left(y^{\prime}\right)-g\left(y^{\prime \prime}\right)\right| \leqq|\beta|\left|y^{\prime}-y^{\prime \prime}\right| /(1-\epsilon|\beta|) \text {. }
$$

Instead of proving differentiability of $g$ at 0 only, we prove differentiability of $g$ at any $y$ in $V$ such that $|y|<\delta / 2|\beta|$, provided $f$ is differentiable at $g(y)$ with differential $\alpha_{1}$. For $y^{\prime}$ and $y^{\prime \prime}$ near to $y$ and within $\delta / 2|\beta|$ of $0, g\left(y^{\prime}\right)$ and $g\left(y^{\prime \prime}\right)$ are within $\delta$ of 0 and near to $g(y)$. For any $\epsilon_{1}>0$, differentiability of $f$ at $g(y)$ implies the relation on $f$ valid for $y^{\prime}, y^{\prime \prime}$ near $y$ :

$$
\left|f g\left(y^{\prime}\right)-f\left(g\left(y^{\prime \prime}\right)\right)-\alpha_{1}\left(g\left(y^{\prime}\right)-g\left(y^{\prime \prime}\right)\right)\right| \leqq \epsilon_{1}\left|g\left(y^{\prime}\right)-g\left(y^{\prime \prime}\right)\right| .
$$

Application of $\beta$ on the left gives:

$$
\begin{array}{r}
\left|\beta\left(f\left(g\left(y^{\prime}\right)\right)\right)-\beta\left(f\left(g\left(y^{\prime \prime}\right)\right)\right)-\beta \alpha_{1}\left(g\left(y^{\prime}\right)-g\left(y^{\prime \prime}\right)\right)\right| \\
\leqq \epsilon_{1}|\beta|\left|g\left(y^{\prime}\right)-g\left(y^{\prime \prime}\right)\right| .
\end{array}
$$

As before, we apply $\beta f g=\beta$ to simplify the first two terms. We write $\beta \alpha_{1}=\beta \alpha+\beta\left(\alpha_{1}-\alpha\right)$ and since $\beta \alpha g=g$, we may replace $\beta \alpha$ by 1 , leading to:

$$
\begin{array}{r}
\left|\beta\left(y^{\prime}-y^{\prime \prime}\right)-\left(1+\beta\left(\alpha_{1}-\alpha\right)\right)\left(g\left(y^{\prime}\right)-g\left(y^{\prime \prime}\right)\right)\right| \\
\leqq \epsilon_{1}|\beta|\left|g\left(y^{\prime}\right)-g\left(y^{\prime \prime}\right)\right| .
\end{array}
$$

Now it was observed in the remarks following the definition of strong differential that $\left|\alpha_{1}-\alpha\right| \leqq \epsilon$, so $\left|\beta\left(\alpha_{1}-\alpha\right)\right|<\epsilon|\beta|<1 / 2$. So $1+\beta\left(\alpha_{1}-\alpha\right)$ has a bounded inverse of norm $\leqq 1 /(1-\epsilon|\beta|)$. Applying $-\left(1+\beta\left(\alpha_{1}-\alpha\right)\right)^{-1}$ to the vector on the left above and changing the order of the terms gives:

$$
\begin{array}{r}
\left|g\left(y^{\prime}\right)-g\left(y^{\prime \prime}\right)-\left[1+\beta\left(\alpha_{1}-\alpha\right)\right]^{-1}\left(\beta\left(y^{\prime}-y^{\prime \prime}\right)\right)\right| \\
\leqq \epsilon_{1}|\beta|\left|g\left(y^{\prime}\right)-g\left(y^{\prime \prime}\right)\right| /(1-\epsilon|\beta|) .
\end{array}
$$

But by (5) the right side may be replaced by $\epsilon_{1}|\beta|^{2}\left|y^{\prime}-y^{\prime \prime}\right| /(1-\epsilon|\beta|)^{2}$, if $y^{\prime}$ and $y^{\prime \prime}$ are near to $y$. Since the coefficient of $\left|y^{\prime}-y^{\prime \prime}\right|$ approaches 0 as $\epsilon_{1} \rightarrow 0, g$ is differentiable at $y$ with differential $\left[1+\beta\left(\alpha_{1}-\alpha\right)\right]^{-1} \beta .^{2}$ In particular, this reduces to $\beta$ at $y=0$.

2 By letting $y^{\prime \prime}=y$ the same proof gives the existence of a Fréchet differential provided $f$ has a Frechet differential at $g(y)$. 
We now prove the third identity $\left(2^{\prime \prime \prime}\right)$. If $y$ is near to 0 , both $y$ and $f(\beta(y))$ are within $\delta / 2|\beta|$ of 0 . We wish to compare $g(f(\beta(y)))$ and $\beta(y)$, which therefore serve as the $x^{\prime}$ and $x^{\prime \prime}$ in this case. The relation about $\beta f$ is:

$$
\begin{array}{r}
|\beta(f(g(f(\beta(y)))))-\beta(f(\beta(y)))-\beta \alpha(g(f(\beta(y)))-\beta(y))| \\
\leqq \epsilon|\beta||g(f(\beta(y)))-\beta(y)| .
\end{array}
$$

Now $\beta f g=\beta$ cancels the first two terms on the left, while $\beta \alpha g=g$ and $\beta \alpha \beta=\beta$ causes the relation to simplify to $|g(f(\beta(y)))-\beta(y)|$ $\leqq \epsilon|\beta||g(f(\beta(y)))-\beta(y)|$, a contradiction unless $g(f(\beta(y)))=\beta(y)$, since $\epsilon|\beta|<1 / 2$.

There remains only to prove uniqueness of $g$; if $g^{\prime}$ has similar properties, then $g^{\prime}(y)=g(y)$ in a neighborhood of 0 . We consider $y$ so near to 0 that $g$ and $g^{\prime}$ satisfy $\left(2^{\prime}\right)$ and $\left(2^{\prime \prime}\right)$, and so that $|g(y)|$ and $\left|g^{\prime}(y)\right|$ are nearer than $\delta$ to 0 in $U$. Then, using the same method as before, we obtain the relation:

$$
\left|\beta(f(g(y)))-\beta\left(f\left(g^{\prime}(y)\right)\right)-\beta \alpha\left(g(y)-g^{\prime}(y)\right)\right| \leqq \epsilon|\beta|\left|g(y)-g^{\prime}(y)\right|
$$

The first two terms cancel, for $\beta(f(g(y)))=\beta(y)=\beta\left(f\left(g^{\prime}(y)\right)\right)$. Then $\beta \alpha g=g$ and $\beta \alpha g^{\prime}=g^{\prime}$, so the expression simplifies to $\left|g(y)-g^{\prime}(y)\right|$ $\leqq \epsilon|\beta|\left|g(y)-g^{\prime}(y)\right|$, and so $g(y)=g^{\prime}(y)$.

REMARK. The equation $\beta \alpha \beta=\beta$ in the hypothesis and the equations $\left(2^{\prime}\right),\left(2^{\prime \prime}\right),\left(2^{\prime \prime \prime}\right)$ are cases where $\alpha \beta, \beta \alpha, f g$ or $g f$ can be cancelled locally from a triple composite mapping $V$ into $U$. The list of identities of this sort is completed with $g \alpha \beta=g$ and $g f g=g$. These may be proved in the same way that $\left(2^{\prime \prime \prime}\right)$ is proved. In contrast, if we propose such an identity for a triple composition from $U$ to $V$, we always have $\beta=0$ as a possible choice of $\beta$, and the resulting $g$ is then 0 . The triple composition then gives 0 , while the cancellation gives $\alpha$ or $f$, which may be different from 0 .

COROLlary. If in particular $\beta \alpha$ is the identity of $U$ or $\alpha \beta$ is the identity of $V$, then correspondingly $g(f(x))=x$ for $x$ near 0 , or $f(g(y))=y$, for y near 0.

Proof. In the first case $g(f(x))=g(f(\beta(\alpha(x))))=\beta(\alpha(x))=x$, using $\left(2^{\prime \prime \prime}\right)$. In the second case, $f(g(y))=\alpha(\beta(f(g(y))))=\alpha(\beta(y))=y$, using $\left(2^{\prime}\right)$.

\section{REFERENCES}

1. T. M. Apostol, Mathematical analysis, Reading, Massachusetts, AddisonWesley, 1957.

2. L. V. Kantorovich, Functional analysis and applied mathematics (translated from the Russian by Curtis D. Benster), National Bureau of Standards Report, 1952.

Case Institute of Technology 